\title{
A tuneable, femtosecond pulse source operating in the range 1.06 - 1.33 microns based on an Yb doped holey fiber amplifier
}

\author{
J.H.V. Price, K. Furasawa, T.M. Monro, L. Lefort, D.J. Richardson \\ Optoelectronics Research Centre \\ University of Southampton \\ United Kingdom \\ jhvp@orc.soton.ac.uk
}

We report soliton pulse formation, amplification and soliton-self-frequency-shifting in an anomalously dispersive, $\mathrm{Yb}$-doped holey fiber amplifier. Our fiber based system provides a highly-practical, continuously-tuneable, femtosecond pulse source operational in the important and difficult to access wavelength range from 1.05 to 1.35 microns. 


\title{
A tuneable, femtosecond pulse source operating in the range 1.06 - 1.33 microns based on an Yb doped holey fiber amplifier
}

\author{
J.H.V. Price, K. Furasawa, T.M. Monro, L. Lefort, D.J. Richardson \\ Optoelectronics Research Centre \\ University of Southampton \\ United Kingdom \\ jhvp@orc.soton.ac.uk
}

Wavelength tunable femtosecond optical pulse sources have applications in areas as diverse as ultrafast spectroscopy, materials processing, optoelectronics, nonlinear optics and optical chemistry. In the past, such sources have been available only in limited wavelength ranges and were realized using solid state lasers with complex, high precision cavities. More recently, the soliton self frequency shift (SSFS) in silica fibers has been exploited to obtain tuneable femtosecond pulses[1]; tuning from 1.55-2.2 $\mu \mathrm{m}$ was previously reported in conventional silica fiber[2], and tuning from 1.3-1.6 $\mu \mathrm{m}$ has been demonstrated using tapered microstructured fiber[3]. Here we report what we believe is the first demonstration of a continously tunable soliton source in the wavelength range $1.06-1.33 \mu \mathrm{m}$, a wavelength range that is difficult to access using conventional solid state laser technology. The system is based on diode pumped $\mathrm{Ytterbium}\left(\mathrm{Yb}^{3+}\right)$ doped silica fiber components, and operates at $\mathrm{mW}$ average input power levels in a $4.7 \mathrm{~m}$ length of fiber.

A schematic of our source is shown in Fig. 1. Ultrashort pulses at $1.06 \mu \mathrm{m}$ are launched into a short length of anomalously dispersive $\mathrm{Yb}^{3+}$ doped holey fiber configured as an amplifier. The pulses rapidly form solitons, and the wavelength is then tuned by SSFS. As described below, by varying the pump power, we were able to adjust the soliton peak power and thus control the magnitude of the SSFS to continously tune the wavelength of the output pulses to wavelengths out as far as $1.33 \mu \mathrm{m}$. Use of an active amplifier medium to provide the pulse compression and shifting is important. For example, it allows us to wavelength shift relatively low energy seed pulses (e.g. from a simple diode pumped fiber oscillator), and it allows us electronically tune the wavelength of the output pulses via control of the amplifier gain. The distributed amplification process also allows us to tune the source over a broader wavelength range than has hitherto been possible with passive tapered fiber devices. With our current system we could obtain femtosecond pulses at wavelengths as long as $1.58 \mu \mathrm{m}$.

The mode locked $1.06 \mu \mathrm{m}$, diode pumped $\mathrm{Yb}^{3+}$ fiber based seed laser souce was constructed by the authors and is reported elsewhere [4]. It produces $60 \mathrm{pJ}$ picosecond pulses ( $\sim 54 \mathrm{MHz}$ repitition rate, $3 \mathrm{~mW}$ average power) selected with a positive linear chirp, which are compresssible externally to $110 \mathrm{fs}$ (using bulk gratings or holey fiber). The chirped pulses are coupled into a holey fiber amplifier which is co-directionally pumped using a $966 \mathrm{~nm}$ diode MOPA (maximum output power $\sim 250 \mathrm{~mW}$ ). We obtain approximately $20 \%$ power coupling into the holey fiber for both the seed pulses and diode pump.

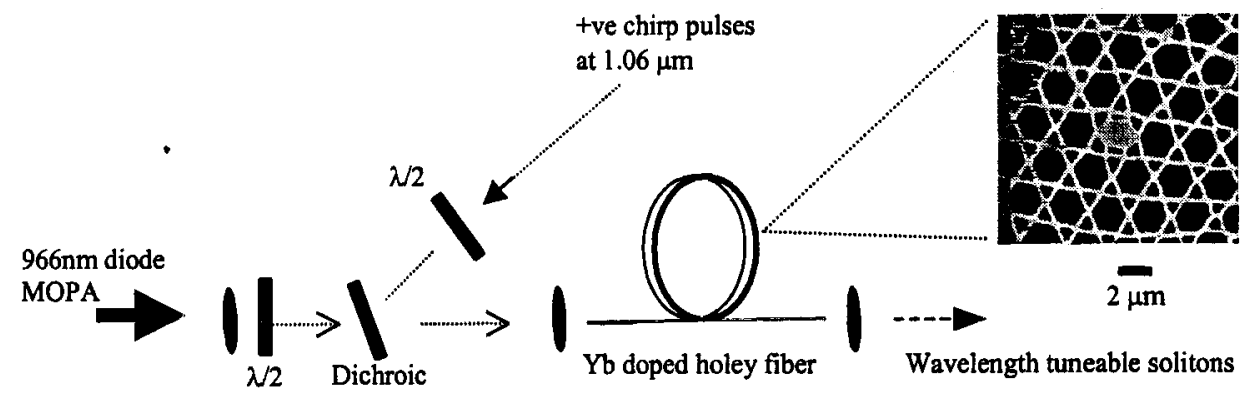

Fig. 1. Experimental configuration. Inset: Scanning electron micrograph of the holey fiber structure with $\mathrm{Yb}^{3+}$ doped core. 
The $4.7 \mathrm{~m}$ length of polarization maintaining, $\mathrm{Yb}^{3+}$-doped holey fiber is shown inset to Fig. 1. The fiber is predicted to have anomalous dispersion from approximately $0.7 \mu \mathrm{m}$ to above $1.8 \mu \mathrm{m}$, and when configured as a laser, it has a slope efficiency of $\sim 60 \%$ [5]. The small, $2 \mu \mathrm{m}$ diameter core, approximately one fifth the size typical in standard fiber, results in a high effective nonlinearity, and short device lengths for SSFS.

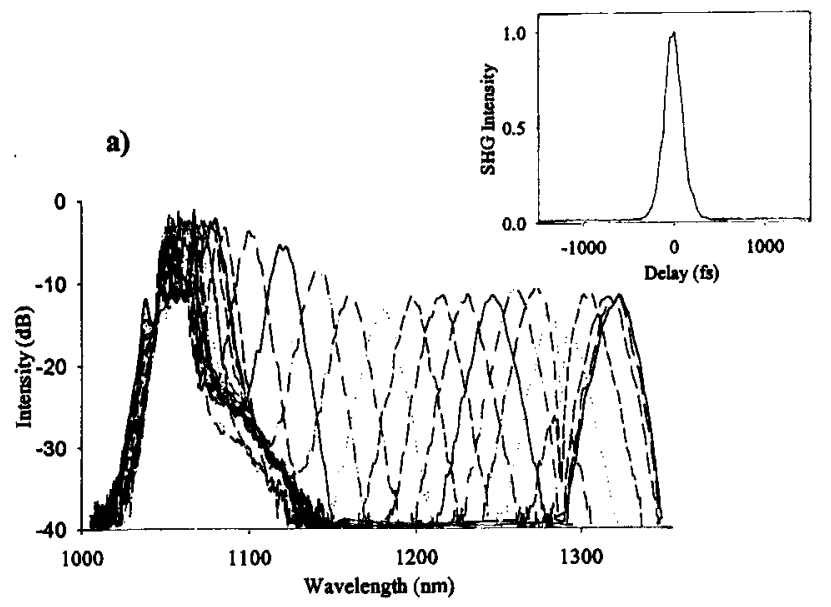

b)

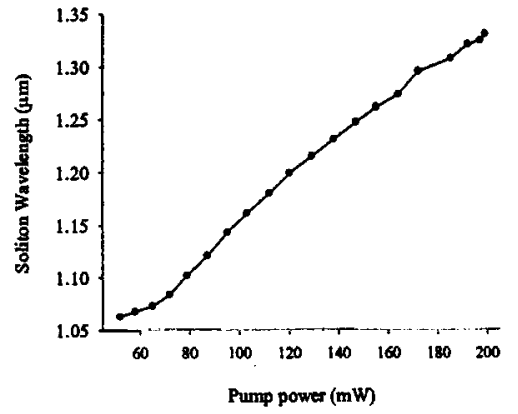

Fig. 2. a) Superimposed spectra of solitons at different wavelengths. Inset: typical autocorrelation of $(1.22 \mu \mathrm{m})$ wavelength shifted soliton; $\tau(\mathrm{FWHM})=180 \mathrm{fs}, \mathrm{b})$ Tuning curve of soliton waveleength vs. amplifier pump power.

As shown in Fig. 2. by increasing the pump power, we obtain continuously tunable soliton pulses from 1.06-1.33 $\mu \mathrm{m}$. Fig. 2a) shows the superimposed spectra of solitons at different wavelengths. Shown inset is the autocorrelation of a typical pulse at a wavelength of $1.215 \mu \mathrm{m}$. Fig. $2 \mathrm{~b}$ ) shows a graph of the soliton wavelength vs. amplifier pump power. Autocorrelation measurements indicate that pulse duration remains approximately constant $\sim 180 \mathrm{fs}$ FWHM as the wavelength is tuned $(\Delta v \Delta \tau \sim 0.6)$.

At increased pump and seed pulse powers, we observe the generation of multicolor pulses (separated in wavelength and time). Pulses with wavlengths out to $1.58 \mu \mathrm{m}$ were obtained. Lastly, we note that with a shorter length of $\mathrm{Yb}^{3+}$ doped amplifier fiber, we obtained pulses compressed to $67 \mathrm{fs}$.

In conclusion, we have developed a continuously tunable soliton source based on diode-pumped active microstructured holey fiber, which we believe to be an exciting development of fiber technology providing a device with great potential for widespread practical applications.

[1] N. Nishizawa, T. Goto, "Compact System of Wavelength-Tunable Femtosecond Soliton Pulse Generation Using Optical Fibers" IEEE Photonics Technology Letters 11, 325-327 (1999).

[2] M.E.Fermann, A. Galvanauskas, M.L. Stick, K.K. Wong, D. Harter, and L. Goldberg, "Ultrawide tunable Er soliton fiber laser amplified in Yb-doped fiber," Opt. Lett. 24, 1428-1430 (1999).

[3] X. Liu, C. Xu, W.H. Knox, J.K. Chandalia, B.J. Eggleton, S.G. Kosinski, and R.S. Windeler, "Soliton self-frequency shift in a short tapered air-silica microstructure fiber," Proc. Conf. Opt. Fiber Comms. (OFC).

[4] J.H.V. Price, L. Lefort, D.J. Richardson, G.J. Spuhler, R.Paschotta, U. Keller, C.Barty, A. Fry, J. Weston, “A practical, low-noise, stretched pulse $\mathrm{Yb}^{3+}$ doped fiber laser," Proc. CLEO 2001.

[5] K. Furasawa, T.M. Monro, J.C. Baggett, P. Petropoulos, P.W. Turner, D.J. Richardson, "A mode-locked ytterbium doped holey fiber laser," Proc. CLEO 2001. 